\title{
Gait Analysis in Cervical Spondylotic Myelopathy
}

\author{
Hirosuke Nishimura, Kenji Endo, Hidekazu Suzuki, Hidetoshi Tanaka, \\ Takaaki Shishido, Kengo Yamamoto
}

Department of Orthopedic Surgery, Tokyo Medical University, Tokyo, Japan

\begin{abstract}
Study Design: Gait analysis of patients with cervical spondylotic myelopathy (CSM) by using a sheet-type gait analysis system.
Purpose: The aim of this study was to compare the gait patterns of patients with CSM, evaluated by the Nurick grades, and to determine the threshold values of gait parameters predicting the occurrence of a fall by using a gait recorder.

Overview of Literature: Gait disorder due to CSM may progress to severe paraplegia, following even a minor trauma such as a fall. The indications for the surgery of CSM without severe paralysis remain controversial. The quantitative gait analysis and the decision for decompressive surgery in patients with CSM are important in order to prevent severe paraplegia from a fall.

Methods: One hundred thirty-two subjects (normal, 34; CSM, 98) underwent gait analysis by using a sensor sheet. Measurements of gait cycle parameters included the step and stride length, step width, foot angle, swing phase, and stance phase. CSM was assessed by Nurick grade.

Results: Although the clinical symptoms were lacking, Nurick grade 1 had significant abnormalities in the parameters of velocity, step length, and step angle $(p<0.05)$. Regarding the Nurick grade and walking phase, the length of the stance phase was increased to more than $70 \%$ of the entire walking cycle in Nurick grade 4.

Conclusions: Gait analysis was an objective tool for evaluating the gait stability. Our results suggested that when the percentage of the stance phase in the gait cycle increases to above 70\%, the CSM patients have an increased fall risk.
\end{abstract}

Keywords: Gait; Movement disorders; Physical examination; Spinal cord diseases

\section{Introduction}

The total population of Japan is predicted to decrease by 2050 , and a substantial increase is expected in the population of age 85 years and above [1]. With the rapid increase in the elderly population, the number of patients with locomotive syndrome has increased in Japan [2,3]. Locomotive syndrome increases the societal burdens with respect to mortality, quality of life, and economic costs. Cervical cord compression was present in $5 \%$ to $7 \%$ of asymptomatic subjects and was associated with physical performances in elderly people. It is suggested that locomotive syndrome involves the early stages of cervical spondylotic myelopathy (CSM). Especially in the elderly people, the gait impairment can be due to CSM which could aggravate the symptoms of cervical cord injury from a relatively minor trauma, such as a fall from a height, or even progress to severe quadriparesis. However, to the best of our knowledge, there are no objective criteria for the evaluation of gait analysis. The number of studies for gait analysis in CSM patients is small, and the indications for the surgery of CSM without severe paralysis still remain controversial [4-9]. Quantitative gait analysis and the decision for decompressive surgery in the patients with CSM are

Received Nov 10, 2014; Revised Nov 28, 2014; Accepted Nov 28, 2014

Corresponding author: Hirosuke Nishimura

Department of Orthopedic Surgery, Tokyo Medical University,

6-7-1 Nishishinjuku, Shinjuku-ku, Tokyo 160-0023, Japan

Tel: +81-3-3342-6111, Fax: +81-3-3342-5295, E-mail: hirosuke819@hotmail.com 
Table 1. Subjects

\begin{tabular}{lccccc} 
Nurick grade & Number & Age (yr) & Height (cm) & Weigt (kg) & Sex (male:female) \\
\hline 0 & 34 & $52.7 \pm 16.5$ & $165 \pm 8.4$ & $59.2 \pm 10.5$ & $18: 16$ \\
1 & 32 & $67.0 \pm 16.9$ & $16 \pm 16.9$ & $64.8 \pm 9.5$ & $32: 0$ \\
2 & 14 & $52.7 \pm 15.9$ & $165 \pm 8.19$ & $59.6 \pm 10.5$ & $14: 0$ \\
\hline 3 & 22 & $58.2 \pm 10.8$ & $167 \pm 7.82$ & $65.6 \pm 8.91$ & $22: 0$ \\
\hline 4 & 30 & $67.0 \pm 16.9$ & $159 \pm 7.54$ & $61.9 \pm 10.6$ & $12: 18$ \\
\hline
\end{tabular}

Values are presented as mean \pm standard deviation.

important, in order to prevent severe paraplegia resulting from a fall. The objective of our study is to compare the gait patterns in patients with CSM, which is evaluated by the Nurick grades, and to determine the threshold values of gait parameters predicting the occurrence of a fall by using a gait recorder.

\section{Materials and Methods}

The participants had a clinical diagnosis of CSM confirmed by magnetic resonance imaging findings. CSM was defined as a constellation of symptoms and signs supported by appropriate radiological findings, including symptoms (numb clumsy hands, impairment of gait, bilateral arm paresthesia) and signs (corticospinal distribution motor deficits, atrophy of hand intrinsic muscles, hyperflexia, positive Hoffman sign, upgoing plantar responses, lower limb spasticity, broad-based unstable gait). One hundred thirty-two subjects (normal, 34; CSM, 98) underwent gait analysis by using a sheet-type gait analysis system. All subjects provided written informed consent after receiving explanation of the experimental protocol, and this study was approved by the Institutional Review Board of our institution. The patient characteristics are summarized in Table 1. Patients with the following conditions were excluded from this study: hip, knee, and ankle joint disease; other spinal disease; and scoliosis of more than $10^{\circ}$. Participants with clear clinical and radiological evidence of CSM were included in this study.

The walking test was performed by using a $2.4 \mathrm{~m}$ long thin-type sensor sheet (Sheet Type Gait Analyzer Walk Way MW-1000, Anima, Tokyo, Japan).

The patients were examined 3 times, and the average of those results were taken. Measurements of gait cycle parameters included the step angle, step length, step width, and gait velocity. Outward rotation of the feet was

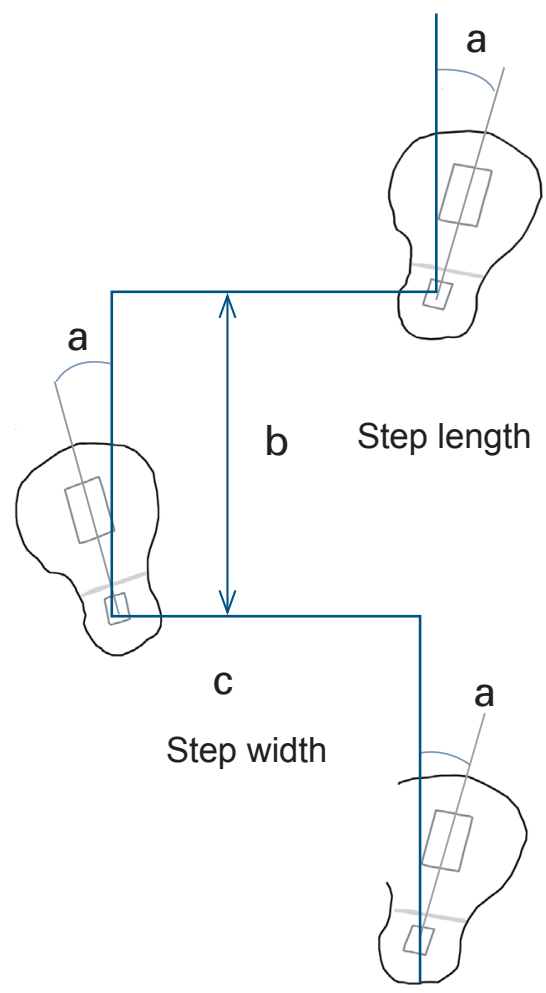

Fig. 1. Definitions of gait parameters.

measured as a positive angle (Fig. 1). The gait cycle consists of a swing phase and a stance phase. Generally, the stance phase and swing phase account for $60 \%$ and $40 \%$, respectively (Fig. 2) [10]. The clinical gait disturbance in patients with CSM was evaluated according to the Nurick grade (Table 2) [11].

A medical statistical consultant performed the statistical analyses using the JMP software package, ver. 8.0 (SAS Institute Inc., Cary, NC, USA). The Tukey-Kramer HSD test was used to analyze the differences in the gait parameters among Nurick grades. The correlations among the variables of gait parameters were examined using the Spearman's rank correlation coefficient. The gait param- 

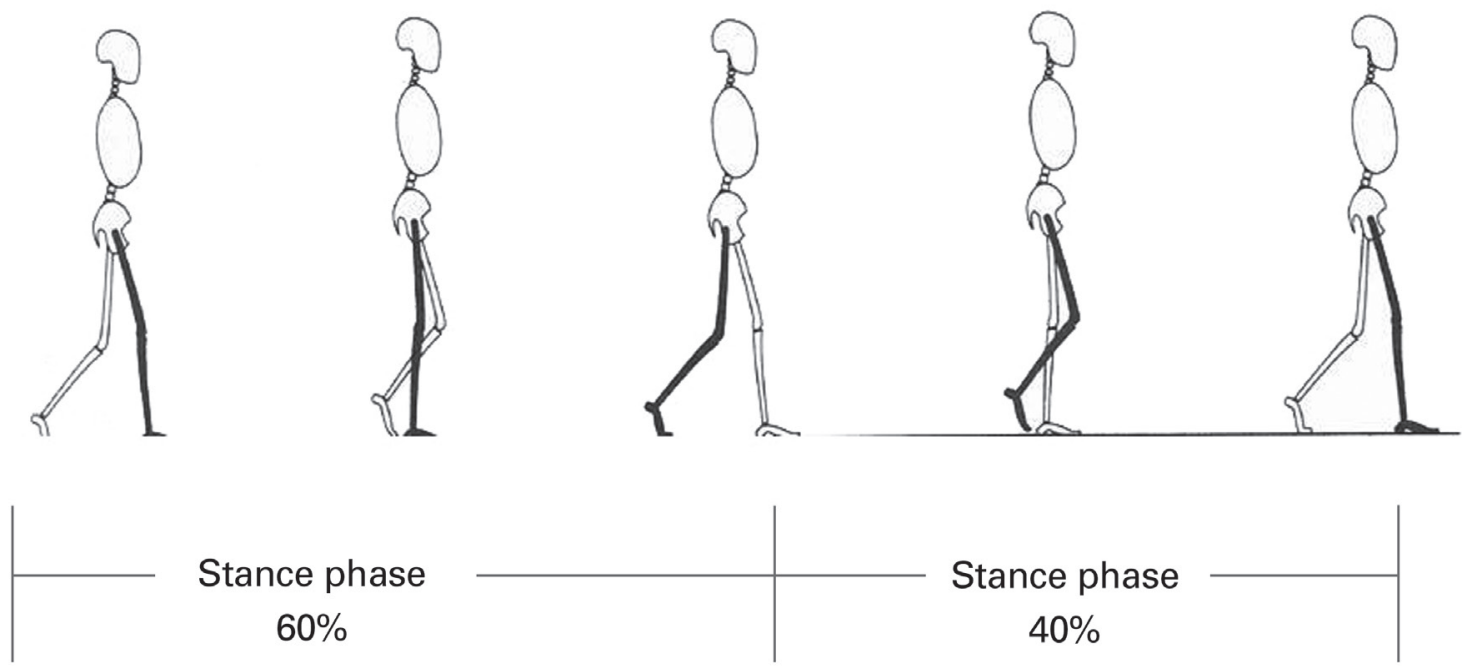

\section{Gait cycle}

Fig. 2. Gait cycle.

Table 2. Nurick grades

\begin{tabular}{ll} 
Grade & \multicolumn{1}{c}{ Description } \\
0 & No evidence of cord involvement \\
1 & Signs of cord involvement normal gait \\
2 & Mild gait involvement able to be employed \\
3 & Gait abnormality prevents employment \\
\hline 5 & Able to ambulate only with assistance \\
\hline
\end{tabular}

Modified from Nurick [11].

eters and risk factors in the patients with Nurick grade 4 were evaluated by logistic regression analysis. A $p$-value of less than 0.05 was considered to indicate a statistically significant difference.

\section{Results}

Gait analysis without any subjective gait disturbance in patents with CSM.

In order to assess the subclinical findings, we compared Nurick grade 0 and 1 . Although clinical symptoms were lacking, grade 1 had significant abnormalities in the parameters of velocity, step length, and step angle $(p<0.05)$ (Fig. 3). The gait velocity and step length decreased as symptoms progressed. In contrast, step angle and step width increased (Fig. 3).

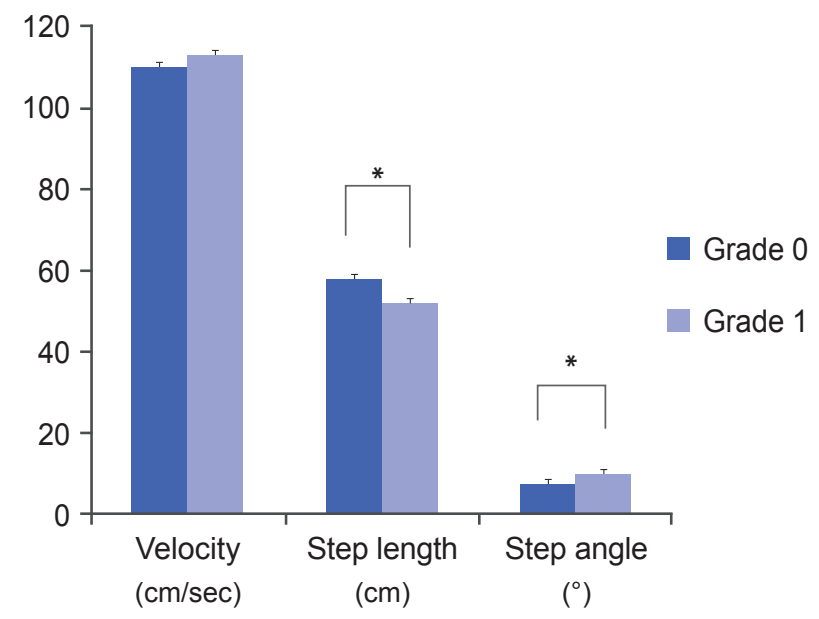

Fig. 3. Nurick grade 0 vs. grade $1 .{ }^{*} p<0.05$

\section{Gait analysis and CSM severity}

For the Nurick grades 1, 2, 3, and 4, the gait parameters were as follows, respectively (Figs. 4, 5): gait velocity $(\mathrm{cm} /$ sec) $107.7 \pm 2.5,94.5 \pm 2.7,92.2 \pm 4.0,76.3 \pm 4.1$; step length (cm) $59.0 \pm 1.2,52.9 \pm 1.3,48.4 \pm 1.7,44.3 \pm 1.7,32.0 \pm 2.9$; step width $(\mathrm{cm}) 7.73 \pm 2.0,9.1 \pm 2.2,9.8 \pm 2.1,11.3 \pm 2.1,12.5 \pm 3.0$; step angle $\left({ }^{\circ}\right) 7.57 \pm 2.1,10.5 \pm 1.6,11.9 \pm 2.1,14.5 \pm 1.6$, $23.5 \pm 2.2$; stance phase (\%) $64 \pm 4.4,67 \pm 4.4,67 \pm 3.4$, $73 \pm 4.4$. When the grade of Nurick classification became worse, the velocity and step length decreased, and the step width and step angle increased (Fig. 4). 
Regarding the Nurick grade and walking phase, the length of the stance phase increased in more than $70 \%$ of the entire walking cycle in the patients with Nurick grade 4. There were no changes in the swing phase (Fig. 5).

\section{The evaluation of fall risk}

We established that grade 4 patients, who were able to ambulate only with assistance, were at the great risk for a fall.

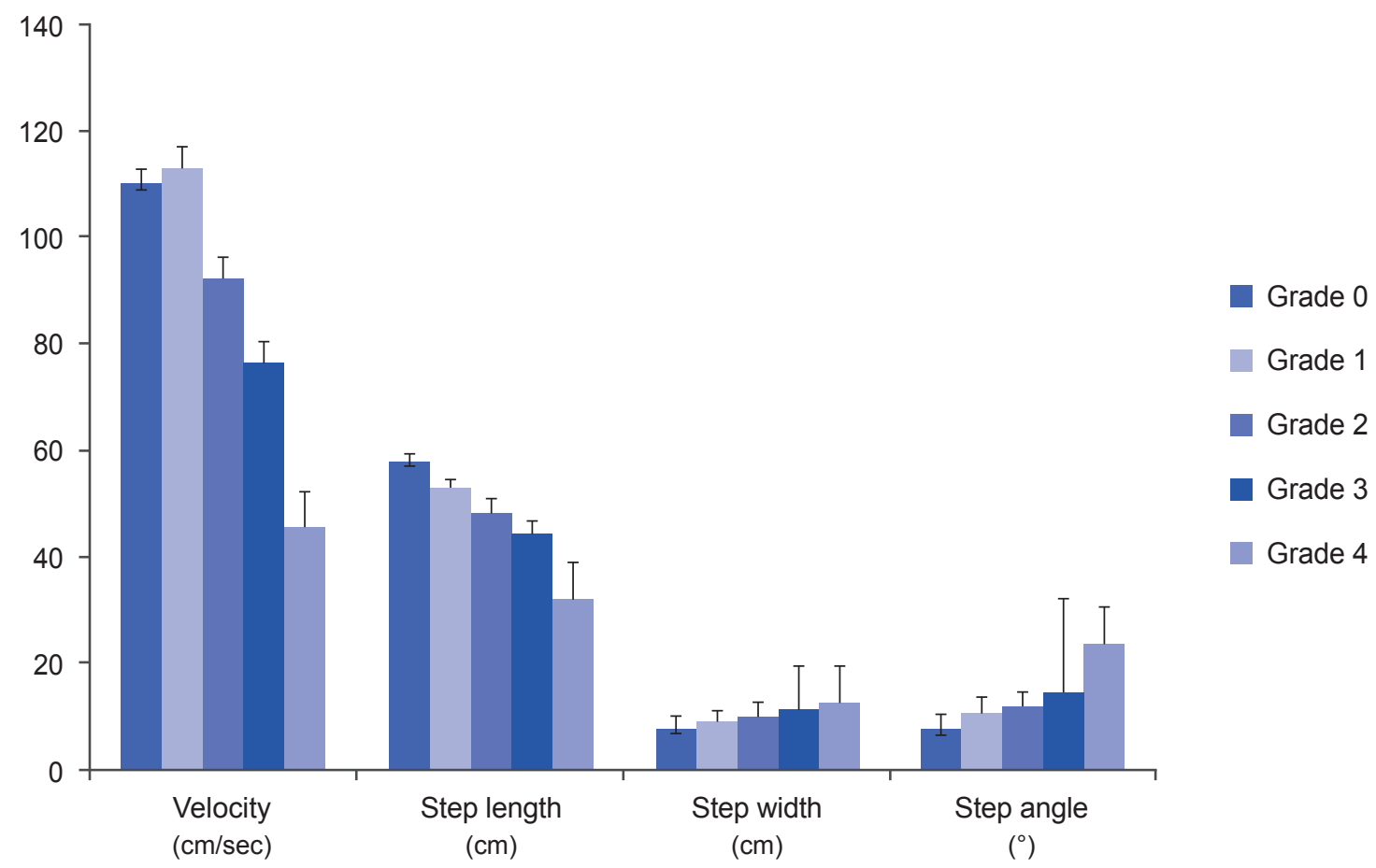

Fig. 4. Nurick grade and gait parameters.

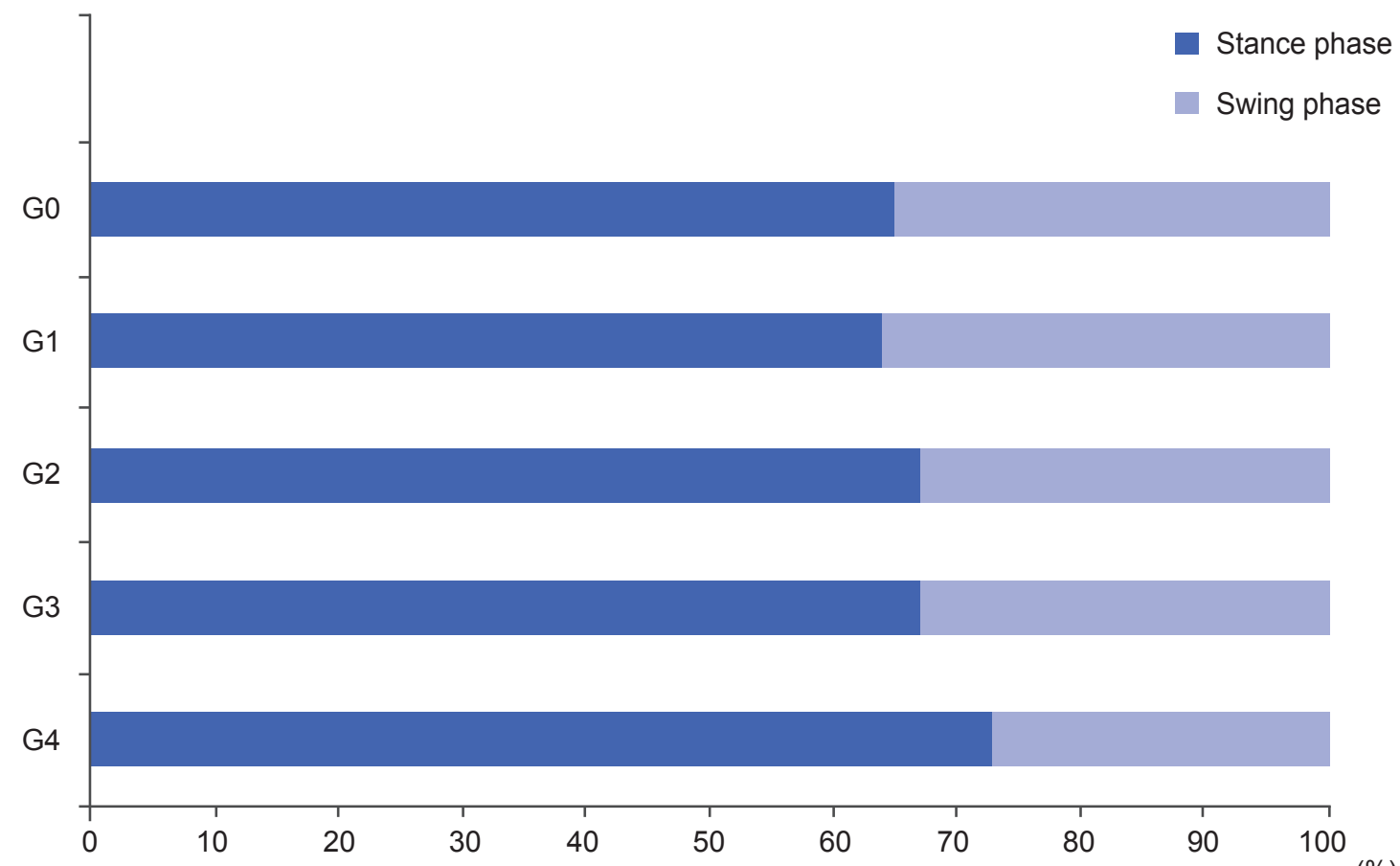

Fig. 5. Nurick grade and walking phase. 
Table 3. Risk factors in Nurick grade 4 patients

\begin{tabular}{lcc} 
& Odds ratio & $p$-value \\
Velocity & 2.8 & 0.001 \\
Step length & 2.1 & $<0.001$ \\
Step width & 1.2 & 0.02 \\
Step angle & 1.3 & $<0.001$ \\
\hline Stance phase & 4.1 & $<0.001$ \\
Swing phase & 0.04 & 0.006 \\
Gait cycle & 1.2 & $<0.001$ \\
\hline
\end{tabular}

Logistic regression analysis showed that the stance phase and swing phase were risk factors for falls for Nurick grade 4 patients (odds ratio=4.1; $p<0.002$.

Logistic regression analysis revealed that the stance phase was a risk factor for falls in the patients with Nurick grade 4 (odds ratio $=4.1 ; p<0.002$ ) (Table 3 ). Our results suggested that the threshold value of the stance phase for increasing risk of falls was likely to be about $70 \%$ of the gait cycle.

\section{Discussion}

The Japanese Orthopedic Association (JOA) has proposed the term "locomotive syndrome". This syndrome is caused by weakening of the musculoskeletal organs such as bones, joints, and muscles [2,3]. It is suggested that locomotive syndrome involves the early stages of CSM. Culhane et al. [12] demonstrated that the falls are a major source of morbidity and mortality in the elderly population. Falls occurring in the patients with CSM are particularly likely to result in a permanent, severe quadriparesis. It was reported that the injury in CSM, associated with ossification of the posterior longitudinal ligament, is a poor prognostic factor; and in 15/156 patients (9.6\%), CSM became worse by even a minor external injury [13].

\section{Gait analysis}

Gait impairment is a primary symptom of CSM. Previous studies have identified the reduction in knee flexion, in the early stages of the disease; and in more severe stages of the disease, decreased ankle plantar flexion at the terminal stance and reduced knee flexion during loading response were found [13]. However, little is known about the specific kinetic and kinematic gait parameters.

\section{Changes in gait parameters without subjective symptoms}

It has been stated that CSM patients have an unstable gait due to spasticity of the gastrocnemius at the time of gait grounding [14]. However, the gait pattern of patients with early stage CSM is unclear. Our results suggested that in CSM patients without subjective symptoms, the gait analysis showed significant changes in the step length, step width, and step angle, as compared with healthy persons. Gait analysis could be reflecting the subclinical conditions.

\section{The differences among gait parameters according to Nurick grade}

When the grade of Nurick classification worsened, the velocity and step length were decreased, and the step width and step angle were increased, so as to maintain a stable walk. The differences among those parameters were remarkable, especially between the grades 3 and 4 (Fig. 4). Regarding the risk factors among gait parameters in the patients with Nurick grade 4, the stance phase was remarkable in the odds ratio. This feature may be an expression of the myelopathy and may serve as a protective mechanism from falls.

\section{The evaluation of fall risk and treatment}

We established that the patients with grade 4, who are able to ambulate only with assistance, are at great risk for a fall. Our results suggested that the threshold value of the stance phase for preventing falls might be about $70 \%$. A recent study has recommended that regarding the timing of operations for CSM, patients with Nurick grade 2 CSM were most likely to improve from surgery [15]. The gait analysis using a sheet-type gait analysis system was a useful tool for catching a gait abnormality in the initial stage of CSM without gait disturbance. The compensation mechanism for unstable gait in the patients with CSM was reduction of the velocity and prolongation of the stance phase by a greater step angle and a smaller step length. A previous paper mentioned that at a self-selected speed, the CSM patients walked slowly, with shorter stride lengths and longer double support durations [4]. When the protective mechanism was beyond control, a fall could occur with a smaller step angle and step width. The extension of stance phase more than $70 \%$ in the total cycle period may be a threshold of falls. This study has some 
limitations. There are some differences between the males and females, in the factors including height and weight. However, these may have little influence on the gait cycle, and more complex measurements (e.q., three dementional analysis) were not performed.

Another limitation of this study was that the investigation of the direct link between gait disorder and the resulting occurrence of actual falls in CSM patients could not be carried out for the obvious ethical reasons. For future research, when gait disorder worsens by the aggravation of spastic paralysis, we should consider the breakdown of these protective mechanisms and facilitate the prevention of falls by implementing safety protocols.

\section{Conclusions}

Gait analysis was an objective tool for evaluating the gait stability, even when the CSM patients had no subjective gait disturbance. The compensation mechanism for unstable gait in the patients with CSM was the reduction of velocity and prolongation of the stance phase by a greater step angle and smaller step length. When the stance phase in the gait cycle increases to above $70 \%$ of the total cycle period, the CSM patients may have an increased fall risk.

\section{Conflict of Interest}

No potential conflict of interest relevant to this article was reported.

\section{Acknowledgments}

We are indebted to the Department of International Medical Communications of Tokyo Medical University for the editorial review of the English manuscript. The authors would like to thank Ms. Yuri Amamizu of the Department of Orthopedic Surgery for the initial English draft.

\section{References}

1. National Institute of Population and Social Security Research. Population projection for Japan [Internet]. Tokyo: National Institute of Population and Social Security Research; 2012 [cited 2015 Apr 21]. Available from: http://www.ipss.go.jp/syoushika/tohkei/ newest $04 / \mathrm{sh} 2401 \mathrm{smm} . \mathrm{html}$.

2. Nakamura K. A "super-aged" society and the "loco- motive syndrome". J Orthop Sci 2008;13:1-2.

3. Nakamura K. The concept and treatment of locomotive syndrome: its acceptance and spread in Japan. J Orthop Sci 2011;16:489-91.

4. Malone A, Meldrum D, Bolger C. Gait impairment in cervical spondylotic myelopathy: comparison with age- and gender-matched healthy controls. Eur Spine J 2012;21:2456-66.

5. Singh A, Crockard HA. Quantitative assessment of cervical spondylotic myelopathy by a simple walking test. Lancet 1999;354:370-3.

6. Lee JH, Lee SH, Seo IS. The characteristics of gait disturbance and its relationship with posterior tibial somatosensory evoked potentials in patients with cervical myelopathy. Spine (Phila Pa 1976) 2011;36:E524-30.

7. Suzuki E, Nakamura H, Konishi S, Yamano Y. Analysis of the spastic gait caused by cervical compression myelopathy. J Spinal Disord Tech 2002;15:519-22.

8. Moorthy RK, Bhattacharji S, Thayumanasamy G, Rajshekhar V. Quantitative changes in gait parameters after central corpectomy for cervical spondylotic myelopathy. J Neurosurg Spine 2005;2:418-24.

9. McDermott A, Bolger C, Keating L, McEvoy L, Meldrum D. Reliability of three-dimensional gait analysis in cervical spondylotic myelopathy. Gait Posture 2010;32:552-8.

10. Murray MP, Drought AB, Kory RC. Walking patterns of normal men. J Bone Joint Surg Am 1964;46:335-60.

11. Nurick $S$. The pathogenesis of the spinal cord disorder associated with cervical spondylosis. Brain 1972;95: 87-100.

12. Culhane KM, O'Connor M, Lyons D, Lyons GM. Accelerometers in rehabilitation medicine for older adults. Age Ageing 2005;34:556-60.

13. Matsunaga S, Nakamura K, Seichi A, et al. Radiographic predictors for the development of myelopathy in patients with ossification of the posterior longitudinal ligament: a multicenter cohort study. Spine (Phila Pa 1976) 2008;33:2648-50.

14. Maezawa Y, Uchida K, Baba H. Gait analysis of spastic walking in patients with cervical compressive myelopathy. J Orthop Sci 2001;6:378-84.

15. Pumberger M, Froemel D, Aichmair A, et al. Clinical predictors of surgical outcome in cervical spondylotic myelopathy: an analysis of 248 patients. Bone Joint J 2013;95:966-71. 